\title{
Analysis of the Dynamic Behavior of a Counterbalance Forklift Truck through Multibody Modelling and Simulation
}

\author{
Marco Pinelli $^{1,2}$, Monica Giovannucci ${ }^{2}, \underline{\text { Alberto Martini }}^{3}$ \\ ${ }^{1}$ School of Engineering \\ University of Bologna \\ V.le del Risorgimento 2 \\ 40136 Bologna, Italy \\ marco.pinelli5@studio.unibo.it \\ ${ }^{2}$ Toyota Material Handling \\ Manufacturing Italy SpA \\ Via Persicetana Vecchia 10, \\ 40132 Bologna, Italy \\ ${ }^{3}$ DIN - Dept. of Industrial Engineering \\ University of Bologna \\ V.le del Risorgimento 2, \\ 40136 Bologna, Italy \\ alberto.martini6@unibo.it
}

\begin{abstract}
This work deals with the analysis of the dynamic response of a counterbalance forklift truck prototype when performing standard verification maneuvers, defined by the manufacturer's testing protocols. The research aims at developing numerical tools based on multibody models to predict accurately the dynamic loads acting on the forklift in the conditions of interest. In particular, this study focuses on a specific test condition, namely the passage on a steel plate obstacle at constant speed, which is one of the most severe maneuvers of the reference cycle in terms of dynamic loads. A model of the complete forklift is developed inside a commercial multibody environment. It takes into account the ground/tire interactions, by means of a simplified nonlinear contanct model, and the load handling assembly dynamics. An experimental campaign is designed and conducted to asses the vehicle behavior when running on the obstacle, by measuring the vibrations of the chassis and of the mast, as well as the forces generated by the mast tilting actuators. The measured data are exploited for model update and validation. The numerical results provided by the updated model show a satisfactory accuracy.
\end{abstract}

Keywords: Virtual Testing, Vehicle Dynamics, Tire-ground Interaction, Experimental Validation, Force Transducers.

\section{INTRODUCTION}

Counterbalance forklift trucks (FLTs) represent a very common equipment for material handling in industrial applications [1]. Usually, the vertical compliance of their suspension systems is mainly provided by tires, since they are not equipped with elastic elements and/or shock absorbers, with the vehicle being supported at three points (namely, the two front wheels and the pivot of the rear axle, which determine the stability triangle). In addition, solid rubber tires or cushion tires are adopted for most applications. These features cause the behavior of FLTs during motion to be significantly affected by ground irregularities, hence safety issues and high dynamic loads possibly being experienced [2-5]. Accordingly, new FLT prototypes manufactured by Toyota Material Handling Manufacturing Italy S.p.A. (Bologna, Italy), which promoted this research, must be verified with rigorous experimental campaigns to assess the actual vehicle response and measure the dynamic stresses that its main components may undergo during operation. Such tests are costly and time consuming, also because numerous transducers (typically strain gauges rosettes) are needed to monitor complex components properly. 
This study aims at developing a multibody model to predict the dynamic loads experienced by a FLT that hits a steel plate obstacle when running in straight line at constant high speed, which is one of the most critical testing conditions in the manufacturer's verification protocols. In particular, the final objective is implementing reliable numerical tools to achieve an accurate estimate of the dynamic stresses acting on the FLT main components (in particular, the chassis), hence possibly reducing the need for experimental tests. To the Authors' best knowledge, most of the studies on FLT dynamics available in the literature dealt with the topic of vehicle stability [2, 6-11] or operator's safety [12-15], whereas only few works aimed at developing predictive models to help the structural and durability design of FLT components $[16,17]$.

A preliminary model had been developed in [5] to assess the possibility of simulating accurately the dynamic response of the chassis assembly, while neglecting the behavior of the load handling assembly, for the FLT operating unloaded. The results therein reported have been exploited to develop a more refined model that takes into account also the load handling assembly dynamics. It allows to estimate the forces acting between the mast and the chassis, as well as to assess both the unloaded and loaded operating conditions. The new model, implemented by using a commercial multibody software package, is here presented.

\section{DESCRIPTION OF THE INVESTIGATED FORKLIFT}

The studied vehicle is an electric FLT characterized by a total mass of about 6 tons and a load capacity of 2.5 tons. The front axle is driven by an electric motor, whereas the steering mechanism is located in the rear axle. The FLT is equipped with two couples of wheels, with a bigger radius for the front axle tires (Fig. 1 - some portions of the image are concealed due to NDA).

The load handling assembly (forks, fork positioner and mast) includes two actuation systems:

- two hydraulic cylinders (referred to as tilt cylinders) act symmetrically between the chassis and the mast (one on each side) and control the mast tilting angle (backward or forward, with respect to the vertical position);

- one further hydraulic cylinder controls the fork positioner lifting (and the mast extension) through a transmission chain.

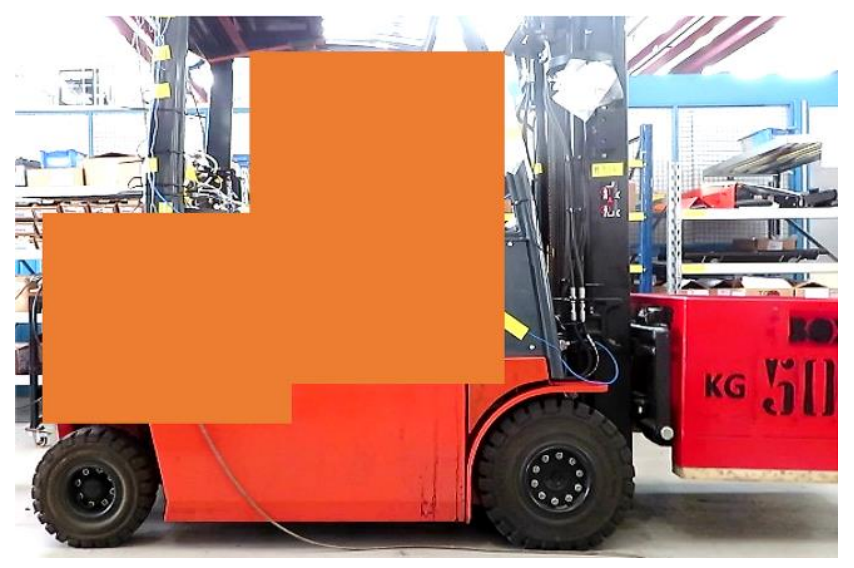

Figure 1. Side view of the studied FLT during tests.

\section{EXPERIMENTAL TESTS}

\subsection{Sensor setup and measurements}

Experiments were performed to characterize the behavior of the studied FLT in both static and dynamic operating conditions. To this purpose, the FLT was equipped with six accelerometers and two load cells.

- Two piezoelectric accelerometers were placed on the left and right sides of the chassis, near the wheel hubs of the front axle, with vertical measuring axis. 
- One piezoelectric accelerometer was placed on the rear part of the chassis, lying on the vehicle vertical-longitudinal plane of symmetry and close to the rear axle pivot, for measuring vertical acceleration as well.

- One triaxial MEMS accelerometer was placed on the chassis under the operator seat, for monitoring the accelerations along the longitudinal, vertical and lateral directions, respectively.

- Two piezoelectric accelerometers were installed on the left and right sides of the mast, above each tilt cylinder joint.

- The two load cells were placed in the mast/tilt cylinders connections, replacing the joint pivots and measuring the axial forces exerted by the tilt cylinders (referred to as tilt forces).

Acceleration and force signals were acquired with a sampling frequency of $5 \mathrm{kHz}$ and $1 \mathrm{kHz}$, respectively. A low-pass filter (cutting frequency $50 \mathrm{~Hz}$ ) has been applied in the post processing to all the signals, since no relevant frequency content was observed above $20 \mathrm{~Hz}$.

Two loading conditions were tested, namely the unloaded FLT (referred to as NL condition) and the FLT loaded at about $80 \%$ of its capacity (WL).

Three different tests were conducted. In the first test, the static tire vertical loads and tilt forces were measured for both the NL and WL conditions, in order to assess the vehicle mass properties and distribution. The ground/tires normal loads were determined by means of vehicle weighing scales lying on a horizontal plane, with the mast in vertical position.

In the second test, the FLT was kept still on the ground and a steel block of $500 \mathrm{~kg}$ was lifted and then rapidly released (Fig. 1). This test was meant to excite primarily the resonance associated with the hydraulic circuit of the tilt cylinders, hence possibly permitting to estimate the corresponding stiffness and damping parameters. The mast was kept tilted by $8^{\circ}$ backwards. Indeed, in this configuration the tilt cylinders are almost orthogonal to the mast, thus making straightforward to determine their equivalent stiffness.

The third test consisted of five passages on the obstacle carried out at constant velocity, for each loading condition. The mast was kept tilted by $8^{\circ}$ backwards (consistently with the test mentioned above), with the forks close to the ground.

\subsection{Experimental results}

The static test showed that the actual mass distribution of the FLT closely matches the nominal one, without significant discrepancies. In particular, the centers of mass of both the load handling assembly and of the chassis assembly lie on the vertical-longitudinal plane of symmetry of the vehicle.

The second experiment appeared successful in exciting the vibration mode involving the oscillations of the mast around its hinges. Figure 2 shows the measured tilt force acting on the right tilt cylinder during the test. Due to NDA, the values are normalized in this chart and in the ones reported hereafter. The oscillations exhibit an exponentially decaying trend, hence being clearly ascribable to a free vibration response. The corresponding damped natural frequency (about $2.5 \mathrm{~Hz}$ ) can be easily determined by computing the Power Spectral Density (PSD) of the signal, while the damping factor can be estimated by using the logarithmic decrement method. These values can be exploited to estimate the equivalent stiffness of the tilt cylinders, by considering the vibrations of a simplified 1-DOF system with known inertia and natural frequency. The estimated stiffness and damping values are used to initialize the numerical model before the updating process.

As for the third test, the measurements exhibited satisfactory repeatability, for each loading condition. Out-of-plane phenomena appear negligible, as the signals measured by the homologous sensors (i.e., on the left and right sides of the FLT) are basically coincident. In particular, the accelerations of the left-front axle (LFA) and the right-front axle (RFA) confirm 
that the front wheels hit the obstacle almost simultaneously, since the maximum delay between the signals is $0.0006 \mathrm{~s}$ (namely, 3 samples).

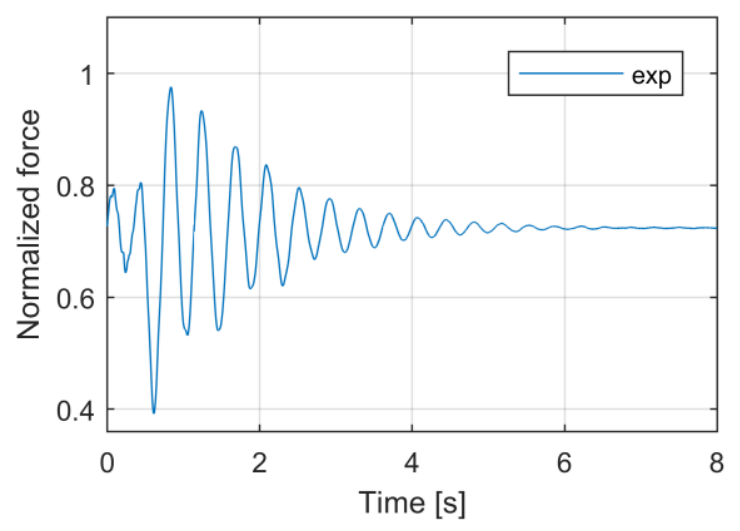

Figure 2. Tilt force measured (exp) on the right tilt cylinder.

Figure 3 shows the analysis in frequency domain of the LFA acceleration signal for the NL loading condition. A major frequency peak at about $5 \mathrm{~Hz}$ that can be observed. By applying a narrow bandpass filter centered on such peak, the front and the rear axle appear in phase. Hence, the frequency is reasonably related to the bounce mode of the vehicle.

Consistently with preliminary investigations [5], the signals measured by the accelerometers on the front axle (particularly the LFA) appear more reliable and with less noise than the others. Therefore, the LFA acceleration is chosen as the reference acceleration signal to be compared with the numerical model.

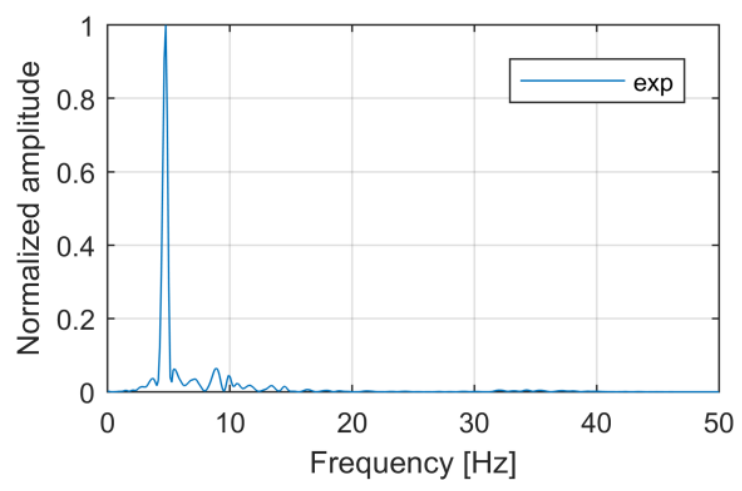

Figure 3. Normalized PSD of the measured (exp) LFA acceleration.

\section{NUMERICAL MODEL AND SIMULATIONS}

\subsection{Model implementation}

A numerical model of the complete FLT (Fig. 4) is implemented by using the multibody software RecurDyn (FunctionBay, Seongnam, South Korea). All the FLT parts are modelled as rigid bodies. Their mass properties are assigned based on both CAD geometries and experimental measurements.

Contact ground-to-surface functions between ground and wheels are set. However, the actual tire compliance is modeled by using a nonlinear lumped-stiffness parameter acting between each wheel and the corresponding axle, estimated from the static load-deflection curve provided by the tire manufacturer.

As for the load handling assembly, the lifting actuator is modeled with as a nonlinear spring that generates a null force when the fork positioner assembly rebounds. The tilt cylinders are modeled with a single linear spring acting on the FLT vertical-longitudinal plane of symmetry: the corresponding initial stiffness and damping values have been estimated as described in 
Section 3.2. The load in the WL condition is a rigid block supported by the forks through contact functions.

All the other joints are modelled by using ideal constraints.

The static vertical loads on the four wheels and the static tilt forces computed with the model closely match the measured ones for both the NL and the WL loading conditions (error below $1.5 \%)$.

Simulations of the vehicle dynamics are performed by prescribing the motion of the driving wheels, through velocity functions.

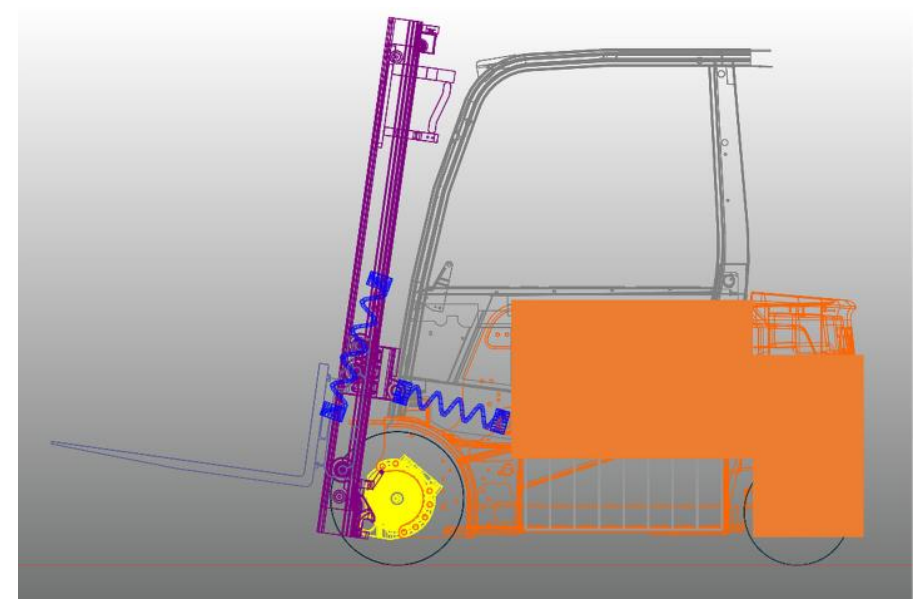

Figure 4. Numerical model implemented with RecurDyn.

\subsection{Model updating and validation}

A preliminary sensitivity analysis permitted to identify the subset of parameters that affect the dynamic response of the FLT most significantly. Such subset was adopted to run the model updating process on the basis of a fully factorial design.

The model accuracy was evaluated in terms of its capability to correctly predict the two measured quantities that are deemed essential for assessing properly the FLT dynamics, according to the manufacturer's knowhow, namely:

- vertical acceleration of the front axle, in terms of amplitude and location of the highest peak (generated by the impact with the obstacle), and of frequency content;

- tilt force, in terms of amplitude and location of the highest peaks, and of frequency content.

Model updating was performed by focusing on the NL case. Indeed, such loading condition is the most critical in terms of accelerations experienced by the FLT, hence possibly causing higher overall stresses.

Figure 5 shows the comparison between the measured data and the numerical results (normalized with respect to the measured maximum value) concerning the NL case, for the LFA acceleration and the tilt force provided by the updated model. It is worth noting that the measured tilt force reported in the graph is the sum of the signals of both load cells, since the tilt cylinders are modelled with a single element. Both quantities are matched satisfactorily by the updated model, in terms of amplitude, main resonance and general damping. The LFA acceleration and tilt force peaks occurring during the obstacle/tires impact phase (at about $0.5 \mathrm{~s}$ ) are slightly overestimated by the simulations. These discrepancies may be reasonably related to local nonlinear deformations of the tires, which are not taken into account by the implemented contact model. Nonetheless the numerical results are deemed sufficiently accurate.

The set of optimal parameters obtained for the NL case were then adopted to simulate also the behavior exhibited by the FLT in the WL case. The comparison between the measured data and 
the numerical results for the WL loading condition, in terms of the LFA acceleration and the tilt force, is reported in Fig. 6. The first peak of both monitored quantities appears largely overestimated. Moreover, some relevant high-frequency oscillations observed in the LFA signal are missed. However, the trend of the tilt force is replicated with sufficient precision. Hence, the accuracy of the model is still considered acceptable, although further improvements may be required.
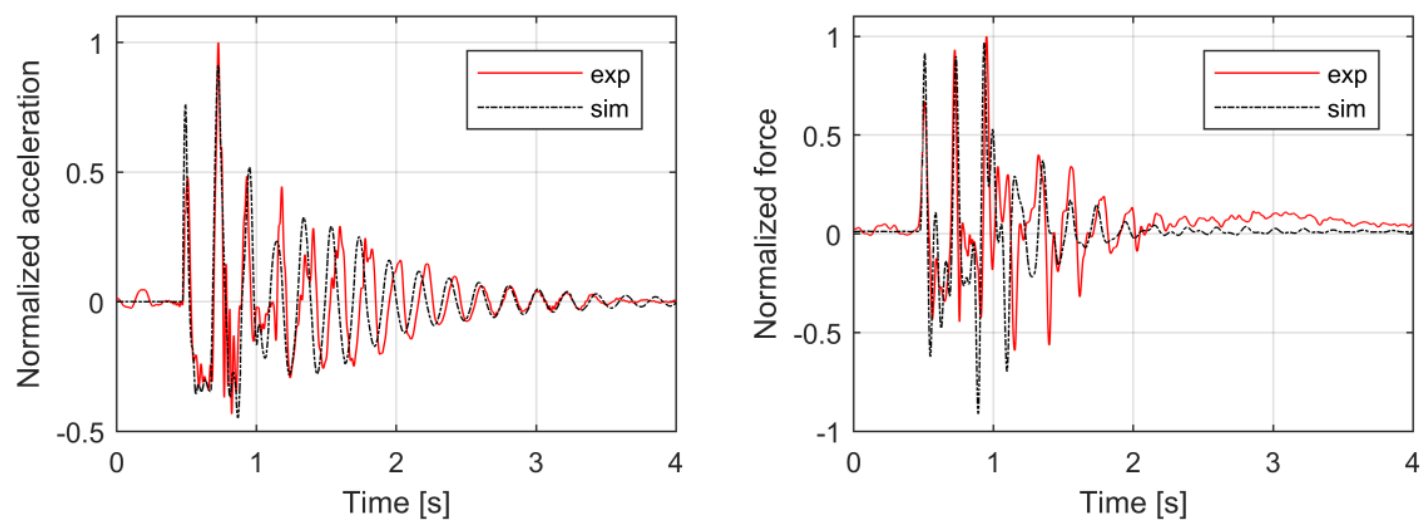

Figure 5. Experimental (exp) vs. numerical (sim) results, NL condition, LFA acceleration (left) and tilt force (right).
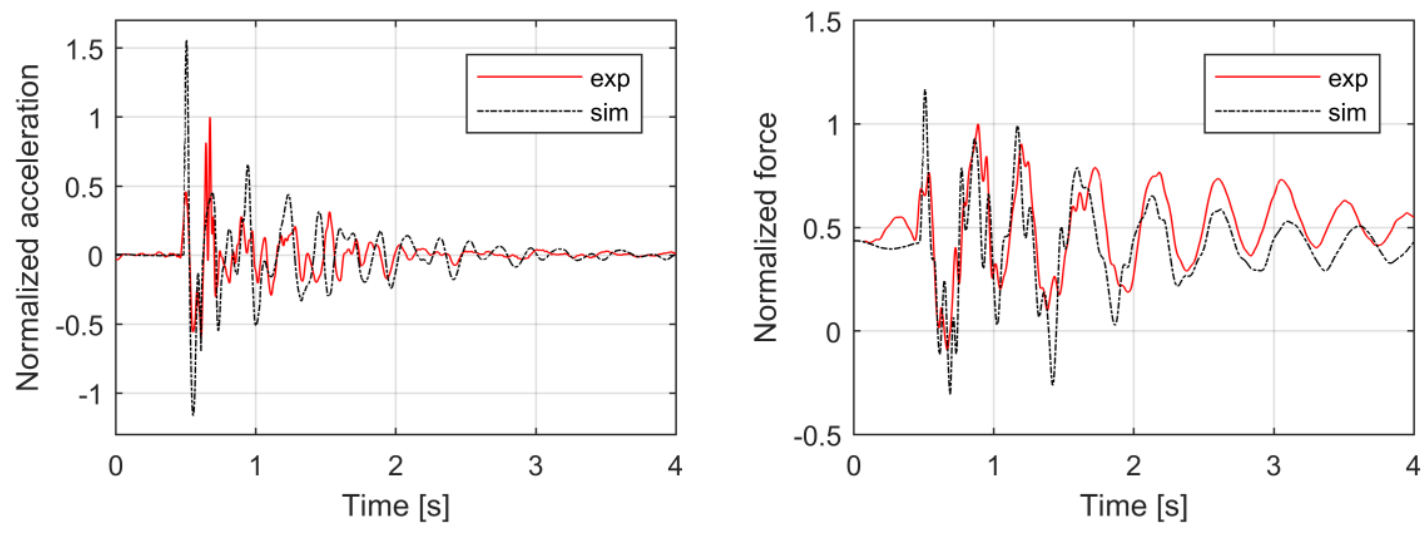

Figure 6. Experimental (exp) vs. numerical (sim) results, WL condition, LFA acceleration (left) and tilt force (right).

\section{CONCLUSIONS}

The presented work investigated the dynamic response of a counterbalance forklift truck executing a reference operating cycle, according to the manufacturer's testing protocols. The study focused on the implementations of numerical tools to predict reliably the forklift behavior in terms of accelerations and forces acting on the chassis, in order to help the structural design process during the development of new products.

An experimental campaign was conducted to characterize the vehicle response when running on a steel plate obstacle at constant velocity with two different loading condition, as well as to estimate the main stiffness and damping properties of the system.

A multibody model of the full vehicle was developed by using a commercial software package. The model takes into account the nonlinear road/wheels interaction and the load handling assembly dynamics. It was updated and validated by exploiting the data gathered from the experiments.

The updated model proven effective in replicating the main dynamic phenomena experienced by the vehicle after impacting the obstacle. In particular, the accelerations and tilt forces exhibited by the unloaded forklift can be closely matched. The numerical results appear acceptable also for the loaded condition, although some discrepancies can be observed for acceleration and tilt 
force peaks related to the impact phase.

The future steps will aim at refining the tire/ground contact model, in order to further improve the model accuracy during the impact phase.

\section{ACKNOWLEDGMENTS}

EnginSoft (Trento, Italy) is gratefully acknowledged for operative cooperation and support.

\section{REFERENCES}

[1] Industrial Truck Association: Lifting America - The Economic Impact of Industrial Truck Manufacturers, Distributors and Dealers, report. Oxford Economics, New York, NY, USA (2017) Available online: https://www.oxfordeconomics.com/recent-releases/lifting-america (accessed on 24th September 2021).

[2] Rebelle, J., Mistrot, P., Poirot, R.: Development and validation of a numerical model for predicting forklift truck tip-over. Vehicle System Dynamics 47(7) (2009) 771-804.

[3] Larsson, T.J., Oldertz, C.: Hazardous exposures and injury types associated with the use of industrial lift trucks in Sweden 2005-2007. Safety Science Monitor 15(3) (2011).

[4] Saric, S., Bab-Hadiashar, A., Hoseinnezhad, R., Hocking, I.: Analysis of forklift accident trends within Victorian industry (Australia). Safety Science 60 (2013) 176-184.

[5] Martini A., Bonelli, G.P., Rivola, A.: Virtual Testing of Counterbalance Forklift Trucks: Implementation and Experimental Validation of a Numerical Multibody Model. Machines 8(2):26 (2020). doi: 10.3390/machines 8020026

[6] Strandberg, L.: Danger, rear wheel steering. Journal of Occupational Accidents 5 (1983) 39-58.

[7] Frazer-Nash Consultancy Limited: Fork lift truck validation and trials, Health and Safety Executive Research Report 456. HSE Books, Sudbury, UK (2006) Available online: http://www.hse.gov.uk/research/rrhtm/rr456.htm (accessed on 24th September 2021).

[8] Popescu, S., Nastase, S., Csatlos, C.: Contributions to theoretical and experimental study of the dynamic stability of the forklift trucks. Journal of Engineering Studies and Research 18(3) (2012) 115121.

[9] Milanowicz, M., Budziszewski, P., Kedzior, K.: Numerical analysis of passive safety systems in forklift trucks. Safety Science 101 (2018) 98-107.

[10] Gardella M., Martini A.: Multibody Models and Simulations to Assess the Stability of Counterbalance Forklift Trucks. In Multibody Dynamics 2019 - ECCOMAS 2019. Computational Methods in Applied Sciences 53 (2020) 526-533. Kecskeméthy, A., Geu Flores, F., Eds.; Springer, Cham, Switzerland. doi: 10.1007/978-3-030-23132-3_63

[11] Larsson, T.J., Lambert, J., Wilde, M., Tully, G., Askew, H., Skinner, W., Carter, B., Martin, T., Kenningham, L.: Industrial forklift trucks - dynamic stability and the design of safe logistics. Safety Science Monitor, 7(1) (2003).

[12] Yang, M., Xu, G., Dong, Q., Han, X.: Vibration Study of Fork-lift Truck Based on the Virtual Prototype Technology. Sensors \& Transducers, 170(5) (2014) 177-183.

[13] Malchaire, J., Piette, A., Mullier, I.: Vibration exposure on fork-lift trucks. Annals of Occupational Hygiene 40(1) (1996) 79-91.

[14] Lemerle, P., Mistrot, P.: A New Tire Model to Predict Vibration Emission of Counterbalance Trucks. Tire Science and Technology 28(2) (2000) 119-137.

[15] De Man, P., Lemerle, P., Mistrot, P., Verschueren, J.-Ph., Preumont, A.: An investigation of a semiactive suspension for a forklift truck. Vehicle System Dynamics 43(2) (2005) 107-119.

[16] Doçi, I., Bajraktari, M., Imeri, V.: Studying dynamic effects on warehouse forklift during forward movement with full loading. In: 16th International Research/Expert Conference Trends in the Development of Machinery and Associated Technology - TMT 2012, Dubai, UAE (2012) 551-554.

[17] Doçi, I., Imeri, V.: Dynamic Analysis of Forklift during Load Lifting using Modeling and Simulations. International Journal of Current Engineering and Technology 3(2) (2013) 342-347. 